\title{
A note on Hedetniemi's conjecture, Stahl's conjecture and the Poljak-Rödl function
}

\author{
Claude Tardif \\ Royal Military College of Canada \\ Canada \\ Claude.Tardif@rmc.ca
}

\author{
Xuding Zhu* \\ Department of Mathematics \\ Zhejiang Normal University \\ Jinhua, Zhejiang, China \\ xdzhu@zjnu.edu.cn
}

Submitted: Jun 12, 2019; Accepted: Oct 28, 2019; Published: Nov 22, 2019

(c) The authors. Released under the CC BY-ND license (International 4.0).

\begin{abstract}
We prove that $\min \{\chi(G), \chi(H)\}-\chi(G \times H)$ can be arbitrarily large, and that if Stahl's conjecture on the multichromatic number of Kneser graphs holds, then we can have $\chi(G \times H) / \min \{\chi(G), \chi(H)\} \leqslant 1 / 2+\epsilon$ for large values of $\min \{\chi(G), \chi(H)\}$.
\end{abstract}

Mathematics Subject Classifications: 05C88, 05C89

\section{Introduction}

The categorical product $G \times H$ of graphs $G$ and $H$ has vertex set $V(G \times H)=\{(x, y)$ : $x \in V(G), y \in V(H)\}$, in which two vertices $(x, y)$ and $\left(x^{\prime}, y^{\prime}\right)$ are adjacent if and only if $x x^{\prime} \in E(G)$ and $y y^{\prime} \in E(H)$. A proper colouring $\phi$ of $G$ can be lifted to a proper colouring $\Phi$ of $G \times H$ defined as $\Phi(x, y)=\phi(x)$. So $\chi(G \times H) \leqslant \chi(G)$, and similarly $\chi(G \times H) \leqslant \chi(H)$. Hedetniemi conjectured in 1966 that $\chi(G \times H)=\min \{\chi(G), \chi(H)\}$ for all finite graphs $G$ and $H$ [6]. The conjecture received a lot of attention [7, 10, 13, 14] and remained open for more than half century. It is known that $\chi(G \times H)=\min \{\chi(G), \chi(H)\}$ whenever $\min \{\chi(G), \chi(H)\} \leqslant 4[1]$ and that the fractional version is true, i.e., for any graphs $G$ and $H, \chi_{f}(G \times H)=\min \left\{\chi_{f}(G), \chi_{f}(H)\right\}$ [15]. However, Shitov refuted this conjecture recently [12]. Yet, some problems concerning the chromatic number of product graphs remain open.

The Poljak-Rödl function $f: \mathbb{N} \rightarrow \mathbb{N}$ is defined by

$$
f(n)=\min \{\chi(G \times H): \chi(G), \chi(H) \geqslant n\} .
$$

*Supported by NSFC 11971438, ZJNSF LD19A010001 and 111 project of the Ministry of Education of China. 
Hedetniemi's conjecture is equivalent to the statement that $f(n)=n$ for all $n$. Shitov proved that for sufficiently large $n, f(n) \leqslant n-1$. Still, very little is known about the behavior of the function $f(n)$. In particular, it is unknown whether $f(n)$ is bounded by a constant. However it is known that if $f(n)$ is bounded by a constant, then $f(n) \leqslant 9$ for all $n$ (see $[10,14]$ ). In this note, we prove the following facts.

\section{Proposition 1.}

(i) $\lim _{n \rightarrow \infty}(n-f(n))=\infty$,

(ii) If Stahl's conjecture on the multichromatic number of Kneser graphs [11] holds, then $\limsup _{n \rightarrow \infty} f(n) / n \leqslant 1 / 2$.

Proposition 1 (i) will be proved in Section 2. Proposition 1 (ii) will be proved in Section 3, where a presentation of Stahl's conjecture is also given.

\section{Discussion and extensions of Shitov's results}

For a positive integer $c$, the exponential graph $K_{c}^{H}$ has vertices all the mappings $f$ : $V(H) \rightarrow\{1,2, \ldots, c\}$, in which $f, g$ are adjacent in $K_{c}^{H}$ if $f(u) \neq g(v)$ for every edge $e=u v$ of $H$. It is well known and easy to verify that $\Phi(v, f)=f(v)$ is a proper $c$ colouring of $H \times K_{c}^{H}$. Thus the way to find counterexamples to Hedetniemi's conjecture is to find an integer $c$ and a graph $H$ such that both $H$ and $K_{c}^{H}$ have chromatic number larger than $c$.

The lexicographic product $G[H]$ of $G$ and $H$ is the graph with vertex set $V(G[H])=$ $\{(x, y): x \in V(G), y \in V(H)\}$, in which two vertices $(x, y)$ and $\left(x^{\prime}, y^{\prime}\right)$ are adjacent if and only if $x x^{\prime} \in E(G)$, or $x=x^{\prime}$ and $y y^{\prime} \in E(H)$.

Shitov's construction of counterexamples to Hedetniemi's conjecture is based on the following result.

Theorem 2 ([12], Claim 3). For any graph $G$ with girth at least six, for all but finitely many values of $q$, we have $\chi\left(K_{c}^{G\left[K_{q}\right]}\right) \geqslant c+1$, with $c=\lceil 3.1 q\rceil^{1}$.

Finding such a lower bound on chromatic numbers of some exponential graphs was the key part of Shitov's refutation of Hedetniemi's conjecture. Finding lexicographic products $G\left[K_{q}\right]$ with $\chi\left(G\left[K_{q}\right]\right)>c$ is standard theory. Indeed the fractional chromatic number $\chi_{f}(H)$ of a graph $H$ is a standard lower bound for its chromatic number, and it is well known that $\chi_{f}(G[H])=\chi_{f}(G) \chi_{f}(H)$ (see [3]). Erdös' classic probabilistic proof [2] shows that there are graphs with girth at least 6 and fractional chromatic number at least 3.1. For such a graph $G$, we have $\chi\left(G\left[K_{q}\right]\right) \geqslant\left\lceil\chi_{f}\left(G\left[K_{q}\right]\right)\right\rceil=\left\lceil\chi_{f}(G) \cdot q\right\rceil \geqslant\lceil 3.1 q\rceil$, and by Theorem 2 , this yields a counterexample to Hedetniemi's conjecture.

Remarkably, replacing the condition $\chi_{f}(G) \geqslant 3.1$ by $\chi_{f}(G) \geqslant B$ for $B \gg 3.1$ readily gives counterexamples to Hedetniemi's conjecture where the chromatic number of at least

\footnotetext{
${ }^{1}$ Technically, Shitov refers to the "strong product" rather than the lexicographic product of graphs, but with $K_{q}$ as a second factor, the strong product coincides with the lexicographic product (see [4]).
} 
one factor is arbitrarily larger than the chromatic number of the product. Also, the proof of Theorem 2 only uses a small subgraph of $K_{c}^{G\left[K_{q}\right]}$. Therefore it is possible that Shitov's construction already gives examples that show that $\lim _{n \rightarrow \infty} f(n) / n=0$. On the other hand, since $\chi_{f}\left(G\left[K_{q}\right]\right)>c$, the fractional version of Hedetniemi's conjecture [15] implies that $\chi_{f}\left(K_{c}^{G\left[K_{q}\right]}\right)=c$. Thus it is also reasonable to think that $\chi\left(K_{c}^{G\left[K_{q}\right]}\right) / c$ may be bounded, and that the identity $\lim _{n \rightarrow \infty} f(n) / n=0$, if true, can only be witnessed by a different construction.

Proof of Proposition 1 (i). Fix a positive integer $d$. We shall prove that if $n$ is sufficiently large, then $f(n+d) \leqslant n$. Let $G_{d}$ be a graph with girth at least 6 and fractional chromatic number at least $8 d$. Then by Theorem 2, for sufficiently large $q$ and $c=\lceil 3.1 q\rceil$, we have $\chi\left(K_{c}^{G_{d}\left[K_{q}\right]}\right) \geqslant c+1$ while $\chi\left(G_{d}\left[K_{q}\right]\right) \geqslant 2 c d$. Now consider the graph $K_{c d}^{G_{d}\left[K_{q}\right]}$. For $i=0,1, \ldots, d-1$, let $Q_{i}$ be the subgraph of $K_{c d}^{G_{d}\left[K_{q}\right]}$ induced by the functions with image in $\{i c+1, i c+2, \ldots, i c+c\}$. Each $Q_{i}$ is isomorphic to $K_{c}^{G_{d}\left[K_{q}\right]}$ and hence at least $c+1$ colours are needed for each copy. For $i \neq j$, each function in $Q_{i}$ is adjacent to each function in $Q_{j}$. Hence, $\chi\left(K_{d c}^{G_{d}\left[K_{q}\right]}\right) \geqslant d(c+1)$. As $\chi\left(K_{d c}^{G_{d}\left[K_{q}\right]}\right)=d c$ and $\chi\left(G_{d}\left[K_{q}\right]\right) \geqslant 2 c d \geqslant c d+d$, it follows that $f(d c+d) \leqslant d c$.

Thus for every $d$ there exist infinitely many values of $n$ (of the form $d c+d$ ) such that $n-f(n) \geqslant d$. It only remains to show that the gap between $n$ and $f(n)$ will not close while going from one value of $c$ to the next. Note that $c=\lceil 3.1 q\rceil$, where $q$ is any value above a fixed threshold, and $\lceil 3.1(q+1)\rceil-\lceil 3.1 q\rceil \leqslant 4$. Thus it suffices to examine the values $n=d c+d+i$ where $i \leqslant 4 d$, and we can suppose that $c \geqslant 5$. The graph $K_{c d+i}^{G_{d}\left[K_{q}\right]}$ contains a copy of $K_{c d}^{G_{d}\left[K_{q}\right]}$ induced by the functions with image in $\{1,2, \ldots, c d\}$. For $j=c d+1, c d+2, \ldots, c d+i$, the constant functions $g_{j}$ with image $j$ are pairwise adjacent and each is adjacent to all the functions in $K_{c d}^{G_{d}\left[K_{q}\right]}$. Hence $\chi\left(K_{c d+i}^{G_{d}\left[K_{q}\right]}\right) \geqslant \chi\left(K_{c d}^{G_{d}\left[K_{q}\right]}\right)+i \geqslant$ $c d+d+i$. For $i \leqslant(c-1) d$, we also have $\chi\left(G_{d}\left[K_{q}\right]\right) \geqslant c d+d+i$, so that $f(c d+d+i) \leqslant c d+i$. Altogether, the inequality $f(n+d) \leqslant n$ is established for all but finitely many values of $n$. Thus, $\lim _{n \rightarrow \infty} n-f(n)=\infty$.

The gap between $n$ and $f(n)$ proved in this section depends on the minimum number $p$ of vertices of a girth 6 graph with fractional chromatic number at least $8 d$. The best known upper bound for $p$ to our knowledge is $p=O\left((d \log d)^{4}\right)$, which follows from a result of Krivelevich [8]. Using this result, one can show that for any $\epsilon>0$, there is a constant $a$ such that for sufficiently large $n, f(n) \leqslant n-a(\log n)^{1 / 4-\epsilon}$. Very recently, He and Wigderson [5] proved that for some $\epsilon \simeq 10^{-9}, f(n)<(1-\epsilon) n$ for sufficiently large $n$. The examples are again cases of Shitov's construction.

\section{Stahl's conjecture}

In the proof of Proposition 1(i), based on the fact that $\chi\left(K_{c}^{G_{d}\left[K_{q}\right]}\right) \geqslant c+1$, we have shown that $\chi\left(K_{c d}^{G_{d}\left[K_{q}\right]}\right) \geqslant c d+d$. In this section, we show that if a special case of a conjecture 
of Stahl on the multichromatic number of Kneser graphs is true, then $\chi\left(K_{c d}^{G_{d}\left[K_{q}\right]}\right)$ is much larger.

Consider a proper colouring $\phi$ of the graph $K_{c d}^{G_{d}\left[K_{q}\right]}$ with $x$ colours. Let $A$ be a subset of $\{1, \ldots, c d\}$ of cardinality $c$. Let $R_{A}$ be the subgraph of $K_{c d}^{G_{d}\left[K_{q}\right]}$ induced by the functions with image contained in $A$. Then $R_{A}$ is isomorphic to $K_{c}^{G_{d}\left[K_{q}\right]}$, so $\phi$ uses at least $c+1$ colours on $R_{A}$. Let $\psi(A) \subseteq\{1, \ldots, x\}$ be a subset of exactly $c+1$ colours used by $\phi$ on $R_{A}$. We have $\psi(A)$ disjoint from $\psi(B)$ whenever $A$ is disjoint from $B$, because $R_{A}$ is totally joined to $R_{B}$ in $K_{c d}^{G_{d}\left[K_{q}\right]}$. This property can be formulated in terms of homomorphisms of Kneser graphs. Recall that the vertices of the Kneser graph $K(m, n)$ are the $n$-subsets of $\{1, \ldots, m\}$, and two of these are joined by an edge whenever they are disjoint. Thus the colouring $\phi: K_{c d}^{G_{d}\left[K_{q}\right]} \rightarrow K_{x}$ induces a homomorphism $\psi: K(c d, c) \rightarrow K(x, c+1)$. The question is how large does $x$ need to be for such a homomorphism to exist.

Stahl's conjecture deals with the latter question. For an integer $n$, the $n$-th multichromatic number $\chi_{n}(H)$ of a graph $H$ is the least integer $m$ such that $H$ admits a homomorphism to $K(m, n)$. In particular $\chi_{1}(H)=\chi(H)$. Lovász [9] proved that $\chi_{1}(K(m, n))=\chi(K(m, n))=m-2 n+2$. Stahl [11] investigated the general multichromatic numbers of Kneser graphs, and observed the following.

a. For $1 \leqslant k \leqslant n, \chi_{k}(K(m, n))=m-2(n-k)$,

b. $\chi_{k n}(K(m, n))=k m$,

c. $\chi_{k+k^{\prime}}(K(m, n)) \leqslant \chi_{k}(K(m, n))+\chi_{k^{\prime}}(K(m, n))$.

Based on this he conjectured the following.

Conjecture 3 ([11]). If $k=a n+b, a \geqslant 1,0 \leqslant b \leqslant n-1$, then for $m \geqslant 2 n$,

$$
\chi_{k}(K(m, n))=\chi_{a n}(K(m, n))+\chi_{b}(K(m, n))=(a+1) m-2(n-b) .
$$

Proof of Proposition 1 (ii). For a fixed $d$, let $G_{d}$ have girth at least 6 and fractional chromatic number at least $8 d$. For any $q$ above a given threshold $q_{d}$ and for $c=\lceil 3.1 q\rceil$, we have $\chi\left(G_{d}\left[K_{q}\right]\right) \geqslant 2 c d$ and $\chi\left(K_{c d}^{G_{d}\left[K_{q}\right]}\right) \geqslant \chi_{c+1}(K(c d, c))$, as explained in the first three paragraphs of this section. If Stahl's conjecture holds, then $\chi\left(K_{c d}^{G_{d}\left[K_{q}\right]}\right) \geqslant 2 c d-2 c+2$. Since $f$ is monotonic, this gives $f(2 c d-2 c+2) \leqslant c d$. Therefore

$$
n \in[2(c-4) d-2(c-4)+2,2 c d-2 c+2] \text { implies } \frac{f(n)}{n} \leqslant \frac{c d}{2(c-4) d-2(c-4)+2} .
$$

The intervals $[2(c-4) d-2(c-4)+2,2 c d-2 c+2], c \in \mathbb{N}$ cover all but a finite part of $\mathbb{N}$. Hence

$$
\limsup \frac{f(n)}{n} \leqslant \lim _{c \rightarrow \infty} \frac{c d}{2(c-4) d-2(c-4)+2}=\frac{d}{2 d-2} .
$$

Since this holds for arbitrarily large $d, \lim \sup \frac{f(n)}{n} \leqslant \frac{1}{2}$. 


\section{Acknowledgements}

We thank Yaroslav Shitov for many helpful comments.

\section{References}

[1] M. El-Zahar, N.W. Sauer, The chromatic number of the product of two 4-chromatic graphs is 4, Combinatorica 5 (1985) 121-126.

[2] P. Erdős Graph theory and probability, Canadian J. Math. 11 (1959), 34-38.

[3] G. Gao, X. Zhu, Xuding, Star-extremal graphs and the lexicographic product, Discrete Math. 152 (1996), 147-156.

[4] W. Imrich, S. Klavžar, Product graphs. Structure and recognition, Wiley-Interscience Series in Discrete Mathematics and Optimization. Wiley-Interscience, New York, 2000. xvi+358 pp.

[5] X. He, Y. Wigderson, Hedetniemi's conjecture is asymptotically false, arXiv:1906.06783v2, 2019.

[6] S. Hedetniemi, Homomorphisms of graphs and automata, Technical Report 0310544-T, University of Michigan, 1966.

[7] S. Klavžar, Coloring graph products - a survey, Discrete Math. 155 (1996) 135-145.

[8] M. Krivelevich, Bounding Ramsey numbers through large deviation inequalities, Random Struct. Algorithms 7 (2) (1995) 145-155.

[9] L. Lovász, Kneser's conjecture, chromatic number, and homotopy, J. Combin. Theory Ser. A 25 (1978), 319-324.

[10] N. Sauer, Hedetniemi's conjecture - a survey, Discrete Math. 229 (2001) 261-292.

[11] S. Stahl, n-tuple colorings and associated graphs, J. Combinatorial Theory Ser. B 20 (1976), no. 2, 185-203.

[12] [12] Y. Shitov, Counterexamples to Hedetniemi's conjecture, Ann. of Math. (2) 190 (2019), no. 2, 663-667.

[13] C. Tardif, Hedetniemi's conjecture, 40 years later, Graph Theory Notes N. Y. 54 (2008) 46-57.

[14] X. Zhu, A survey on Hedetniemi's conjecture, Taiwanese J. Math. 2 (1998) 1-24.

[15] X. Zhu, The fractional version of Hedetniemi's conjecture is true, European J. Combin. 32 (2011), 1168-1175. 